\title{
Diagnosis of Pre- and Post-treatment of Echinococcus granulosus with Counter current Immune Electrophoresis and Bacterial Co-agglutination
}

\author{
Omer Hassan Siddig Elamin ${ }^{1}$, Mai Abdalla Ali ${ }^{2}$, Ibrahim Alhag Almahadi Elgezuli ${ }^{3}$, Mona Alrasheed Bashir ${ }^{4}$ \\ Ivan Kahwa ${ }^{5,6}$ \\ ${ }^{1}$ Department of Microbiology., Faculty of Medicine, University of Khartoum, Sudan \\ ${ }^{2}$ Department of Biochemistry and Molecular Biology, Faculty of Science, University of Gezira, Sudan \\ ${ }^{3}$ Faculty of Health and Environmental Sciences, University of Gezira, Sudan \\ ${ }^{4}$ Department of Biotechnology, Faculty of Science and Technology, Omdurman Islamic University, Sudan \\ ${ }^{5}$ Department of Pharmacy, Faculty of Medicine, Mbarara University of Science and Technology, Mbarara, Uganda \\ ${ }^{6}$ Pharm-Biotechnology and Traditional Medicine Centre of Excellence (ACEII), Mbarara, Uganda
}

\begin{abstract}
Objectives: Echinococcosis is a cyclozoonotic disease caused by E. granulosus. The canine mainly the dog represents the definitive host and immunity plays an important role in the control of the disease. This study was aimed to detect Echinococcus granulosus B-antibody for Dot-ELISA and antigen for counter-current immune electrophoresis (CIEP) and bacterial co-agglutination assay (Co-A).
\end{abstract}

Methods: Follow-up of 23 surgical and chemotherapy patients from different hospitals of Khartoum were performed.

Results: Results: Sensitivity and specificity for Dot-ELISA was $94.4 \%$ and $100 \%$, respectively within seven days to 6 months except for the relapse all or most of the cases were negative for the disease whereas the sensitivity of CIEP and Co-A for Echinococcus granulosus antigen in patient sera was $77.8 \%$ and $94.4 \%$ respectively with a specificity of $100 \%$ and $75 \%$ respectively. Dot-ELISA, showed high positive and negative predictive value (100\%, 95.2\%), respectively with CIEP $(100 \%, 83.3 \%)$ and in Co-A $(81 \%, 94.1 \%)$.

Conclusion: Conclusion: Therefore, detection of antigen of E. granulosus in the serum of operative, post-operative, or chemotherapeutic treated patient using CIEP and Co-A or detection of antibodies using IgG ELISA to the antigen Brich fraction in (ELISA) assay is useful for following treated hydatid patients. J Microbiol Infect Dis 2021; 11(2):88-94.

Keywords: Echinococcus granulosus, Dot-ELISA, Co-agglutination assay, immune electrophoresis

\section{INTRODUCTION}

Echinococcosis or Hydatidosis is an important cyclozoonotic parasite disease caused mainly by two species of the parasite Echinococcus granulosus and Echinococcus multilocularis which belongs to class cestode. Echinococcus granulosus has a cosmopolitan distribution with the dog being the main definitive host and a wide range of intermediate hosts including mammals e.g. ruminants, lagomorphs, artiodactyls, and man. The disease can cause morbidity and economic problems [1] and its development determined by the radiologist [2].
Currently, nine genetically distinct populations (Genotype) assigned G1 to G9 which differ in host range, developing rate, infectivity to human's pathogenicity had been identified in $E$. granulosus [3].

One of the characteristics of human hydatid disease is the frequent relapses that occur after surgery. This makes follow-up of the patients necessary for years after surgery to detect the appearance of new cysts as soon as possible [4].

Correspondence: Mai Abdalla Ali, Department of Biochemistry and Molecular Biology, Faculty of Science, University of Gezira, Sudan

Email: maiabdalla222@gmail.com

Received: 26 June 2020 Accepted: 16 Mai 2021

Copyright (C JMID / Journal of Microbiology and Infectious Diseases 2021, All rights reserved 
After surgery or chemotherapy, it is often difficult to be sure whether the patient is cured of hydatid disease or remains infected. This is a recognized problem in the management of hydatid disease, and is due to the persistence of small or ectopic cysts in other sites even after surgery, the possibility of secondary Echinococcosis following surgery of hydatid cysts, or the development of resistance of cysts to chemotherapy [5]. The aim of the present study is to evaluate serological tests that might differentiate between infected and cured cases of hydatid disease circulating hydatid antigen $(\mathrm{CAg})$ profiles were determined in both pre-and post-operative or chemotherapy sera- countercurrent immune electrophoresis (CIEP) and bacterial co-agglutination assay (Co-A) tests were employed to detect CAg and Dot-ELISA to detect circulating hydatid antibody in the serum.

\section{METHODS}

\section{Blood collection for serum}

During the period of May 2013 to May 2014, 23 patients with hydatid disease drawn from Ibn Sina, Soba, and Khartoum Hospitals had their blood drawn into $5 \mathrm{ml}$ disposable blood containers. From these samples' sera were separated into labeled sterile containers and refrigerated at $-20^{\circ} \mathrm{C}$.

\section{Group 1: Patients treated by surgical operations}

This consisted of six new cases of operated on for hydatid cysts. A single pre-operative serum sample was collected from each patient. After surgery, a minimum of one serum sample was collected from these patients at 3 days, 7 days, 1 month, 6 months, or 1 year. In addition to these cases, seven old cases of hydatid diseases that were operated upon 2 years previously were also followed up and included in the study. A single postoperative serum sample was collected from each case.

\section{Group 2: Patients treated by chemotherapy}

This consisted of five cases of ultrasoundproven hydatid disease and five cases of presumptive hydatid disease. These cases were treated by chemotherapy using albendazole and mebendazole. After chemotherapy, sera were collected from these patients after 6 months and 1 year respectively. The sera were stored at 20o $\mathrm{C}$ for further use. The sera were screened for $\mathrm{Ag}$ and $\mathrm{Ab}$ profiles; CIEP and $\mathrm{Co}$ - $\mathrm{A}$ were employed to detect Ag however, Dot - ELISA for Ab of E. granulosus.

\section{Collection of crude Hydatid antigen and preparation of IgG ELISA to the antigen B- rich fraction antigen}

Crude antigen was collected from camel hydatid liver and lung fertile cyst fluid which was then centrifuged at 1500 r.p.m. for 10 minutes and the supernatant used as crude antigen. The concentration was estimated by Biuret method and adjusted to be between 1-3 $\mu \mathrm{g} / \mu \mathrm{l}$ by adding phosphate buffer saline at a $\mathrm{PH}$ of 7.2 in accordance with a method described by [6] with slight modification. The crude antigen was heated for 10 minutes in a water bath as estimated by [6]. The protein concentration was estimated by Biuret method and was adjusted to be 1-2 $\mu \mathrm{g} / \mu \mathrm{l}$ to make the IgG ELISA to the antigen $\mathrm{B}$-rich fraction solution.

\section{Preparation and Purification of Hyper Immune Antiserum}

Camel hydatid fluids were given $0.3 \mathrm{ml}$ in four limbs of the rabbits each $3-4 \mathrm{~kg}$ in weight. After 10 days, blood samples were taken and monitored for hydatid antibodies to camel hydatid fluid antigen by Dot-ELISA blood collected when the titer of antibodies was 3200 . Hyperimmune antiserum containing hydatid antibodies was purified by the method described by [7].

\section{Dot-enzyme-linked immunosorbent assay (Dot-ELISA)}

$5 \mu \mathrm{l}$ of the IgG ELISA to the antigen B-rich fraction antigen was dotted on nitrocellulose membrane discs and allowed to air dry thoroughly and placed against the binding site in microtitration plate wells. The Dot-ELISA test was done by the method described earlier by [8].

\section{Counter-current-immunolectrophoresis (CIEP)}

This test was performed with hyperimmune hydatid sera on a glass slide containing Bactoagar (Difco) 1\% as described by [5].

\section{Staphylococcus aureus (Cowan's strain I) bearing protein A (SAPA)}

Staphylococcus aureus cells were grown on Mueller-Hinton agar at $37^{\circ} \mathrm{C}$ and fixed with 
formalin. SAPA cells were sensitized and Bacterial Co-agglutination (Co-A) test conducted as described by [25].

\section{RESULTS}

The results of serum antibody diagnosis of hydatid disease of followed up treated patients with Dot- ELISA test results are shown below in Table 1.

Sera from the 23 patients were tested using DotELISA. All the 10 pre-chemotherapy sera were positive for the antibody. However, after one year of chemotherapy, 1 serum still tested positive for Dot-ELISA hence showing 100\% sensitivity. After one year of surgery, 5 sera tested positive before any treatment with chemotherapeutic drugs. One serum was tested negative after seven days of surgery in the first year. Three and two sera tested negative after 1 month and 6 months respectively. It is again identified that during the pre-chemotherapy, in a period of 6 months, 5 sera tested negative and after one year, 4 sera negative as well. It is having been identified in Table 1 that, after 2 years of surgery, 2 sera tested positive.

The results for detection of the hydatid antigen using both CIEP and Bacterial Co-A are shown in Table 2.

In Table 2, it was demonstrated that sera of all the six and four new cases out of the four cases after 1 year of surgery but before treatment was detected by the Co-A and CIEP respectively. The Co-A test demonstrated 1 serum, three sera, 1 serum negatively on the seventh day, one month respectively, and detected 1 serum positively after one year. The CIEP test also detected 4 sera negatively collected on the third day after treatment. After 2 years of surgery, the CIEP and Co-A tests detected two sera and four sera positively, respectively. During prechemotherapy, both CIEP and Co-A tests detected positively eight and nine sera out of the ten new cases. After a period of 6 months, CIEP and Co-A showed a negative detection of 7 sera and 5 sera respectively. However, after a year, CIEP and Co-A showed positive detection of 1 serum and 2 sera, respectively. The sensitivity and specificity results of the three test assays are shown in Table 3.

The Dot- ELISA detected true positive cases (17 out of 18) indicating a $94.4 \%$ sensitivity and all true negative cases indicating a $100 \%$ specificity. Whereas the positive number detected by Co-agglutination and Countercurrent immune electrophoresis tests were 17 and 14 indicating sensitivities of $94.4 \%$ and $77.8 \%$ followed by Co-agglutination $75 \%$ and Counter-current immune electrophoresis $100 \%$

The positive and negative predictive values of Dot-ELISA were higher than other tests $100 \%$ and $95.2 \%$, respectively, while Counter-current immune electrophoresis $100 \%$ and $83.3 \%$ respectively, and Co-agglutination $81 \%$ and $94.1 \%$, respectively.

\section{DISCUSSION}

Echinococcosis/hydatidosis is highly endemic in sub-Saharan Africa including Ethiopia, Kenya, Mauritania, Sudan, and Tanzania [9]. In Sudan, several studies documented the endemicity of cystic Echinococcosis (CE) in different parts of the country [10-14]. Therefore, effective CE control programs show that prevention of transmission to either intermediate or defensive host can reduce or even eliminate the infection in human, and livestock population. Therefore, if either or both hosts can be vaccinated, the effect will be to improve and more rapidly expedite control [15].

Table 1. Dot- ELISA test for serum antibody of hydatid disease of followed up treated patients.

\begin{tabular}{|c|c|c|c|c|c|c|c|}
\hline Patient groups & $\begin{array}{c}\text { Before } \\
\text { Treatment }\end{array}$ & $\begin{array}{c}3 \\
\text { days }\end{array}$ & 7 days & 1 month & 6 months & 1 year & 2 years \\
\hline 1 year after surgery & +5 & & -1 & -3 & -2 & & \\
\hline 2 years after surgery & & & & & & & +2 \\
\hline Pre-chemotherapy & +10 & & & & -5 & -4 & \\
\hline
\end{tabular}


Table 2. Counter -current immune electrophoresis and Bacterial Co-agglutination test for detection of hydatid antigen.

\begin{tabular}{lcccccccc} 
Patient groups & Test & $\begin{array}{c}\text { Before } \\
\text { Treatment }\end{array}$ & 3 days & 7 days & 1 month & 6 months & 1 year & 2 years \\
\hline \multirow{2}{*}{$\begin{array}{l}1 \text { year after surgery } \\
\text { CIEP }\end{array}$} & +4 & -4 & & & & \\
& Co-A & +6 & -1 & -3 & -1 & +1 & \\
2 years after surgery & CIEP & & & & & & +2 \\
& Co-A & & & & & +4 \\
Pre-chemotherapy & CIEP & +8 & & -7 & +1 & +2 \\
\hline
\end{tabular}

Table 3 Sensitivity and specificity of Dot-ELISA, Counter - current immune electrophoresis and Bacterial Co-agglutination test assay

\begin{tabular}{lcccccc}
\hline Test & $\begin{array}{c}\text { No. of positive } \\
\text { cases }\end{array}$ & $\begin{array}{c}\text { Sensitivity } \\
\%\end{array}$ & $\begin{array}{c}\text { No. of Negative } \\
\text { cases }\end{array}$ & $\begin{array}{c}\text { Specificity } \\
\%\end{array}$ & $\begin{array}{c}\text { Positive } \\
\text { predictive value }\end{array}$ & $\begin{array}{c}\text { Negative } \\
\text { predictive value }\end{array}$ \\
\hline $\begin{array}{l}\text { Dot- ELISA } \\
\text { Counter }\end{array}$ & 17 & $94.4 \%$ & 0 & $100 \%$ & $100 \%$ & $95.2 \%$ \\
$\begin{array}{l}\text { current } \\
\text { Co- }\end{array}$ & 14 & $77.8 \%$ & 0 & $100 \%$ & $100 \%$ & $83.3 \%$ \\
agglutination & 17 & $94.4 \%$ & 4 & $75 \%$ & $81 \%$ & $94.1 \%$ \\
\hline
\end{tabular}

After surgery or chemotherapy, it is often difficult to be sure whether the patient was cured of hydatid disease or remains infected. This is the recognized problem in the management of hydatid disease, and is due to the persistence of small or ectopic cysts in other sites even after surgery, the possibility of secondary Echinococcosis following surgery of hydatid cysts, or the development of resistance of cysts to chemotherapy [25]. Therefore, this study was carried out to differentiate between infected and cured cases of hydatid disease.

ELISA has been found to be highly sensitive and suitable for antibody detection of cystic hydatid disease. According to the study of [16], the ELISA was positive in all hydatid cases irrespective of the site of cyst indicating $100 \%$ sensitivity [17]. reported that the sensitivity, specificity, and diagnostic efficacy of detection of free $8-\mathrm{kDa}$ and $16-\mathrm{kDa}$ circulating antigen in acid-treated serum samples was $100 \%$ by ELISA. The study [18] indicated that by employing an antigen from hydatid fluid, retained by Con A sephorase (GP) in ELISA the sensitivity and specificity of the test reached $100 \%$ and $88 \%$, respectively. However, by the immunoblotting method, the antigen showed a sensitivity of $95 \%$ as well as showing higher specificity of up to $100 \%$. Meanwhile, [19] reported that ELISA with $83 \%-95 \%$ sensitivity was more sensitive than other serological methods in hydatid cyst diagnosis. The modification of standard ELISA known as DotELISA, which has been employed later in the diagnosis of human hydatidosis, was found to be very convenient for field studies and poorly equipped diagnostic laboratories $[8,20-23]$. The results were also similar to those of other workers indicating that the Dot-ELISA is very sensitive and specific in detecting antiechinococcal antibodies. [21] showed that the crude antigen has a sensitivity of $97 \%$ and a specificity of $52 \%$ while the B- antigen had a sensitivity of $94 \%$ and a specificity of $90.3 \%$. Cross-reactivity of B- antigen in [21] was mainly found in human cysticercosis and $E$. multilocularis infection. Using serum CAg profiles, no marked differences were between the circulating hydatid antibody detection using 
B-rich protein ELISA of the pre-and posttreatment sera of both surgical and chemotherapeutic treatment sera of both surgical and chemotherapeutic cases of hydatid disease. Detection of 50 and $30 \mathrm{kDa}$ proteins in serum by Dot-ELISA gave a sensitivity of $92.9 \%$ and a specificity of $95 \%$ using B-antigen [24].

The Dot-ELISA [21] reported a sensitivity of $94 \%$ and specificity of $90.5 \%$ as a field test for the diagnosis of hydatid disease in the Turkana region of North West Kenya. In Egypt, [22] reported a sensitivity of $88 \%$ and specificity of $96.9 \%$ by ELISA for demonstration of antibodies in 18 patients with hydatid disease and 32 blood donor controls. The same authors have also evaluated the Dot-ELISA for the detection of circulating hydatid antigen in the serum by using anti-echinococcal hyper-immune rabbit sera. The sensitivity of the test, however, was relatively low (55.6\%), which was attributed to the low volume of circulating antigen. The serologic sensitivity (63\%) for IgG4 antibodies of native $\mathrm{AgB}$ fraction from human hydatid cyst fluid was $74 \%$ sensitivity and $88 \%$ specificity for human CE and 93\% sensitivity and 65\% specificity for native $\mathrm{AgB}$.

Using Dot-ELISA B-rich antigen for detection of circulating hydatid antibody, $63 \%$ of the antigen reacted with IgG4 causing successful follow-up of surgical and treated patient of E. granulosus cyst because IgG4 decrease rapidly after cure patient of $E$. granulosus. Two cases of old surgical patients show positive reactions; this may be due to secondary Echinococcosis or undetectable cyst. Follow-up new surgical cases show negative titer start from day 7 , one case; 6 months, 3 cases; one year, 1 case. This reflects the usefulness of that test in following up surgical and treated patients and also effectiveness in the detection of new cases. Counter-current immune electrophoresis and Co-agglutination are two tests that have been standard and evaluated to demonstrate $\mathrm{CAg}$ in serum for the diagnosis of hydatid disease. Counter-current immune electrophoresis is a highly specific test (100\% specificity) and no false-positive reactions were observed with any sera from the disease patients or healthy controls. The other test is moderately sensitive $(95 \%)$ but less specific (84\%) than countercurrent immune electrophoresis. False-positive reactions were observed with sera from patients with various other parasitic diseases. Both tests are simple, inexpensive, and rapid with results obtainable within 1 hour after receipt of serum. These two tests have a potential after wider application in the serum diagnosis of hydatid disease in poorly equipped laboratories in developing countries [25].

The demonstration of circulating hydatid antigen is useful in the post-treatment evaluation of cases Nos 10 and 11. In this study, a total of 16 pre-operative or pre-chemotherapeutic sera collected from six new cases of surgically treated hydatid disease and ten cases of postsurgically treated hydatid disease and ultrasound suspended patient treated with chemotherapy were screened for the presence of $\mathrm{CAg}$ by both counter-current immuneelectrophoresis and Co-agglutination. Of these, 15 and 12 sera were positive for CAg by Co-agglutination and counter-current immunoelectrophoresis, respectively. The postoperative sera collected from these cases did not show any CAg by the counter-current immune electrophoresis. However, CAg could be detected by Co-agglutination in three or four of these sera collected 3 and 7 days after surgical removal of the cyst respectively. The CAg level in the sera showed a marked decline by the seventh day after surgical removal of the cyst. CAg could not be detected by Coagglutination in the sera collected 1 month after surgery and 6 months after chemotherapy.

Serum samples from seven old cases of hydatid disease (patients who were operated upon 2 years previously), were tested for the presence of Cag; of these seven sera tested all except two were negative for CAg by both counter-current immunoelectrophoresis and co-agglutination tests. The clinical presentations of these two cases, sera of which were positive for the antigen, showed that the infection was still persistent with signs of active clinical disease. This could be due to recurrence of the disease by secondary Echinococcosis or perhaps persistent and undetectable small hydatid cysts were also in the body [5].

\section{Conclusion}

This study recommended that detection of antigen of $E$. granulosus in the serum of operative, post-operative, or chemotherapeutic treated patient using such tools as counter- 
current immune electrophoresis and Bacterial Co-agglutination or detection of antibodies using IgG ELISA to the antigen B-rich fraction antigen or detection of $\lg G 4$ antibody must be encouraged to be used in hospitals for following treated hydatid patients.

The finding of this study confirms similar observation on detection of Antibody using IgG ELISA to the antigen B-rich fraction antigen and in a preliminary study on serum CAg profiles before and after surgical removal of hydatid cysts in five cases and also in assessing the status of infection, whether recent or past. In addition to this, the results of the present study suggest that both pre-and post-surgery or chemotherapy estimation of CAg in serum would be useful in monitoring post-operative or chemotherapeutic cases of hydatid disease and to determine whether the patient is cured of hydatid disease or still has an active infection. In addition to that using IgG ELISA to the antigen B-rich fraction antigen Dot-ELISA for detecting $\mathrm{Ab}$ of $E$. granulosus is more confirmatory to and convenient to the result of previous tests.

\section{ACKNOWLEDGMENTS}

Declaration of Conflicting Interests: The authors declare that they have no conflict of interest.

Financial Disclosure: No financial support was received.

\section{REFERENCES}

1. Budke $\mathrm{CM}$, Carabin $\mathrm{H}$, Ndimubanzi $\mathrm{PC}$, et al. $\mathrm{A}$ systematic review of the literature on cystic Echinococcosis frequency worldwide and its associated clinical manifestations. Am J Trop Med Hyg 2013;88(6):1011-1027.

2. Brunetti E, Kern P, Vuitton DA. Expert consensus for the diagnosis and treatment of cystic and alveolar Echinococcosis in humans. Acta Trop 2010;114(1):116.

3. Thompson, R.C.A. and McManus, D. Aetiology: parasites and life-cycles. In: Eckert, J., Gemmel, M.A., Meslin, F-X and Pawłowski, Z.S., (eds.) WHO/OIE Manual on Echinococcosis in Human and Animals: a Public Health Problem of Global Concern. World Organization for Animal Health, 2001, Paris, France, pp. 1-19.

4. Force L, Torres JM, Carrillo A, et al. Evaluation of eight serological tests in the diagnosis of human Echinococcosis and follow-up. Clin Infect Dis 1992 ;15(3):473-480.
5. Shariff GM, Parija SC. Counter-current immunoelectrophoresis test for serodiagnosis of hydatid disease by detection of circulating hydatid antigen. J Microbiol Methods 1991;14(2):71-76.

6. DalimiasI AAH, Madani R, Ghorbankhani $D$, et al . Comparative evaluation of serodiagnostic Techniques in Hydatid Cyst Disease. Arch Razi Ins 2000; 51: 8593.

7. Gottstein B. An immunoassay for the detection of circulating antigens in human Echinococcosis. Am J Trop Med Hyg 1984;33(6):1185-1191.

8. Zheng GY, Zhao RL, Feng XH. Dotimmunobinding assay in the serodiagnosis of human hydatid disease. Am J Trop Med Hyg 1986;35(4):812814.

9. Romig T, Omer RA, Zeyhle E, et al. Echinococcosis in sub-Saharan Africa: emerging complexity. Vet Parasitol 2011;181(1):43-47.

10. Elmahdi IE, Ali QM, Magzoub MMA, et al. Cystic Echinococcosis of livestock and humans in central Sudan. Ann Trop Med Parasitol 2004; 98(5):473-479.

11. Mohammed AA, El-Malik KH. The Epidemiology of Cystic Echinococcosis in Nyala, Southern Darfur State, Sudan .Sud. J. Vet. Res 2000 16:49-53.

12. Osman AMA, Aradaib IE, Ashmaig ALK, et al. Rapid detection of Echinococcus granulosus-complex and specific identification camel genotype (G6) a nested PCR. In Proceeding Echinococcus of the 22nd International Congress of Hydatiology, Athens, Greece .2007: 80-85.

13. Omer RA, Dinkel A, Romig T, et al. A molecular survey of cystic Echinococcosis in Sudan. Vet Parasitol 2010; 169(3-4):340-346.

14. Ibrahim K, Thomas R, Peter K, et al. A molecular survey on cystic Echinococcosis in Sinnar area, Blue Nile state (Sudan). Chin Med J 2011;124(18):28292833.

15. National Center for Health Statistics (US) and National Center for Health Services Research, 1994. Health, United States. US Department of Health, Education, and Welfare, Public Health Service, Health Resources Administration, National Center for Health Statistics.

16. Wattal $\mathrm{CH}$, Malla NA, Khan IA, et al. Comparative evaluation of enzyme-linked immunosorbent assay for the diagnosis of pulmonary Echinococcosis. J Clin Microbiol 1986;24(1):41-46.

17. Kanwar JR, Kaushik SP, Sawhney IM, et al. Specific antibodies in serum of patients with hydatidosis recognised by immunoblotting. J Med Microbiol $1992 ; 36(1): 46-51$.

18. Sbihi Y, Janssen D, Osuna A. Serologic recognition of hydatid cyst antigens using different purification methods. Diagn Microbiol Infect Dis 1996;24(4):205-211. 
19. Babba H, Messedi A, Masmoudi S, et al. Diagnosis of human hydatidosis: comparison between imagery and six serologic techniques. Am J Trop Med Hyg 1994;50(1):64-68.

20. Pappas MG. Recent applications of the Dot-ELISA in immunoparasitology. Vet Parasitol 1988;29(23):105-129.

21. Rogan MT, Craig PS, Zeyhle E, et al. Evaluation of a rapid dot-ELISA as a field test for the diagnosis of cystic hydatid disease. Trans $\mathrm{R}$ Soc Trop Med Hyg1991;85(6):773-777.

22. Romia SA, Youssef ME, Handoussa AE, et al. Dot-ELISA as a diagnostic test in hydatid disease. J Egypt Soc Parasitol 1992;22(3):603-610.

23. Parija SC. A review of some simple immunoassays in the serodiagnosis of cystic hydatid disease. Acta Trop 1998;70(1):17-24.

24. Ramadan MM, El-Ameer AM, Shalash IR, et al. Diagnosis of Echinococcosis (hydatidosis) using dotELISA. Egypt J Hosp Med 2016 ;64(1):304-310.

25. Shariff M, Parija SC. Co-agglutination (Co-A) test for circulating antigen in hydatid disease. $\mathrm{J}$ Med Microbiol 1993; 38(6):391-394. 